\title{
String Cosmology
}

\author{
John D. Barrow and Kerstin E. Kunze \\ Astronomy Centre \\ University of Sussex \\ Brighton BN1 9QJ \\ $\mathrm{UK}$
}

\begin{abstract}
An overview is given of the formulation of low-energy string cosmologies together with examples of particular solutions, successes and problems of the theory.
\end{abstract}

\section{Introduction}

General relativity provides a successful theory to describe classical phenomena in gravity. Several experiments provide impressive evidence for how well it succeeds. The Hulse-Taylor binary pulsar 'PSR1913+16 consists of a pulsating and a non-pulsating neutron star. According to general relativity 'the orbit should slowly decay and the orbital period should increase due to the loss of energy by the 'emission of gravitational waves. This has been indeed observed [1] to very high precision. However, despite this outstanding success, under certain extreme physical conditions general relativity predicts that it cannot predict [2]. We expect general relativity to be a low-energy and low space-time curvature limit of some quantum theory of gravitation that has yet to be found. String cosmology is one approach to discover the cosmological consequences of some of these quantum gravitational corrections to the general theory of relativity.

The search for a unification of quantum field theory and general relativity is the most important quest in theoretical physics. Unfortunately, the canonical quantum field approach to quantum gravity is riddled with incurable infinities and so is not well defined. Superstring theory, in contrast, is a very promising candidate for a consistent theory of quantum gravity [3]. In all, there are five consistent superstring theories, namely, type $I, I I A, I I B, E_{8} \times E_{8}$ heterotic and $S O(32)$ heterotic, which all seem to be related by duality symmetries facilitating the existence of a more fundamental underlying theory. Attempts to identify this underlying M-theory (see, for example, [四) have yet to arrive at a complete final formulation and interpretation.

Due to anomaly considerations, superstring theories are only consistent in ten dimensional spacetimes. In order to make contact with the observed four-dimensional world it is assumed that the extra six spatial dimensions are compactified. For simplicity, it is often assumed that the internal space is a torus. However, particle physics requires the internal space to be more complicated, namely of CalabiYau type [5]. In their low-energy limit string theories predict a classical gravitational interaction. 
This theory of gravity differs from standard Einstein relativity. However, general relativity is a particular solution. In the simplest models, and to lowest order, the corrections to standard gravity come in the form of two fields: the dilaton, which is a scalar field, and the antisymmetric tensor field strength, which is a three-form (see, for example, [6]). In principle, moduli fields from the compactified dimensions should also be taken into account. In four dimensions, the antisymmetric tensor field strength can be described in terms of its (Hodge) dual which is the gradient of a scalar field. So, in the simplest models the gravitational sector of low-energy string theory describes general relativity coupled to two stiff perfect fluids.

Most approaches to string cosmology begin with the low-energy effective action. However, as in general relativity, the low-energy theory suffers from space-time curvature singularities. An interesting example of a regular, inhomogeneous solution is given in [7]. In regions of high curvature and/or string coupling the low-energy limit of string theory can no longer be trusted and higher-order corrections have to be taken into account [8]. However, it is an open question, as to when a solution to the low-energy theory is also an exact solution to all orders or can be extended to one. A notable exception is provided by plane-wave space-times, which are exact solutions to string theory [9]. Other exact solutions corresponding to conformal field theories with a cosmological interpretation are discussed for example in [10].

The inflationary paradigm [11] has become an important extension of the standard big bang model in order to explain certain cosmological puzzles and provide seeds for large-scale structure formation. The implementation of inflation in the low-energy limit of string theory requires some thought since in the simplest models there is no potential for the dilaton, which would be needed for the standard inflationary picture where the evolution of the inflaton is driven by a non-vanishing potential energy density [12]. Any potential (for example, arising from supersymmetry breaking) is too steep to dominate the expansion dynamics and leaves the kinetic energy of the dilaton to dominate [13]. One possibility to implement inflation in string theory was discussed in [14].

Another interesting approach is suggested by the pre-big-bang model [15. Ten-dimensional Minkowski space-time is an exact solution to the full superstring theory. This corresponds to a free theory where the string coupling vanishes $(g \rightarrow 0)$ as the dilaton goes off to minus infinity $(\Phi \rightarrow-\infty)$. The basic assumption of pre-big-bang string cosmologies is that the evolution of the universe did indeed begin near this trivial but exact vacuum of string theory [16].

\section{Low-energy Effective String Theory}

The low-energy effective action compactified to four dimensions is given by [6] [17]

$$
S=\frac{1}{2} \int d^{4} x \sqrt{-g} e^{-\Phi}\left[R+\nabla_{\mu} \Phi \nabla^{\mu} \Phi-\frac{1}{12} H_{\mu \nu \rho} H^{\mu \nu \rho}\right]
$$

where $\Phi$ is the dilaton, $H_{\mu \nu \lambda}$ is the antisymmetric tensor field strength which is defined by

$$
H_{\mu \nu \rho}=\partial_{[\mu} B_{\nu \rho]}
$$

In general, the low-energy effective action can be written in the form of the Einstein-Hilbert action of general relativity in 4 dimensions by applying the conformal transformation of the space- 
time metric $g_{\mu \nu}$ [17]

$$
g_{\mu \nu} \rightarrow \tilde{g}_{\mu \nu}=\exp [-\Phi] g_{\mu \nu}
$$

In the so-called Einstein frame, as opposed to the string frame used above, the action (11) is

$$
S=\frac{1}{2} \int d^{4} x \sqrt{-\tilde{g}}\left[\tilde{R}-\frac{1}{2} \tilde{\nabla}_{\mu} \Phi \tilde{\nabla}^{\mu} \Phi-\frac{1}{12} \exp [-2 \Phi] \tilde{H}_{\mu \nu \rho} \tilde{H}^{\mu \nu \rho}\right] .
$$

Since low-energy string theory results in general relativity coupled to the aforementioned fields in the Einstein frame this frame is often used to find solutions. However, one should keep in mind that the original string frame provides the physical interpretation. The classical equations of motion for a free test string imply that its world-sheet is a minimal surface with respect to the string frame: the analog of the motion of free point particles along geodesics. Furthermore, the characteristic size of a fundamental string is constant in the string frame. So one can describe how, for example, distances between objects vary w.r.t. their intrinsic size and it makes sense to talk about 'small' and 'large' curvature scales [18. Hence, one could argue that strings "see" the string frame metric $g_{\mu \nu}$ rather than the conformally related Einstein frame metric $\tilde{g}_{\mu \nu}$ [19].

In the Einstein frame the equations of motion are given by 17

$$
\begin{aligned}
\tilde{R}_{\mu \nu}-\frac{1}{2} \tilde{g}_{\mu \nu} \tilde{R} & ={ }^{(\Phi)} \tilde{T}_{\mu \nu}+{ }^{(H)} \tilde{T}_{\mu \nu} \\
\tilde{\nabla}_{\mu}\left[\exp (-2 \Phi) \tilde{H}^{\mu \nu \lambda}\right] & =0 \\
\tilde{\square} \Phi+\frac{1}{6} e^{-2 \Phi} \tilde{H}_{\alpha \beta \gamma} \tilde{H}^{\alpha \beta \gamma} & =0
\end{aligned}
$$

where

$$
\begin{aligned}
{ }^{(\Phi)} \tilde{T}_{\mu \nu} & =\frac{1}{2}\left(\Phi_{, \mu} \Phi_{, \nu}-\frac{1}{2} \tilde{g}_{\mu \nu} \tilde{g}^{\alpha \beta} \Phi_{, \alpha} \Phi_{, \beta}\right) \\
{ }^{(H)} \tilde{T}_{\mu \nu} & =\frac{1}{12}\left[e^{-2 \Phi}\left(3 \tilde{H}_{\mu \lambda \kappa} \tilde{H}_{\nu}^{\lambda \kappa}-\frac{1}{2} \tilde{g}_{\mu \nu} \tilde{H}_{\alpha \beta \gamma} \tilde{H}^{\alpha \beta \gamma}\right)\right] .
\end{aligned}
$$

(5) can be rewritten using differential forms as

$$
d *\left(e^{-2 \Phi} \tilde{H}\right)=0
$$

where $*$ is the Hodge operator. Using its properties and those of the exterior derivative $d$, it is found that the antisymmetric tensor field strength can be expressed in terms of a scalar field $b$ :

$$
\tilde{H}^{\mu \nu \lambda}=e^{2 \Phi} \tilde{\epsilon}^{\rho \mu \nu \lambda} b_{, \rho},
$$

where $\tilde{\epsilon}^{\rho \mu \nu \lambda}$ is the totally antisymmetric tensor. Furthermore, there is the Bianchi identity

$$
d \tilde{H}=0 .
$$

The dynamics of $b$ are determined by (11), which yields,

$$
\tilde{\square} b+2 \tilde{\nabla}^{\mu} \Phi \tilde{\nabla}_{\mu} b=0 .
$$




\section{$3 \quad$ Symmetries}

The four-dimensional low-energy action is invariant under global $O(d, d ; \mathbb{R})$ and global $S L(2, \mathbb{R})$ transformations, where $d$ is the dimension of the compactified space.

\section{1 $O(d, d ; \mathbb{R})$ Symmetry from Dimensional Reduction}

In 20] it is shown that dimensional reduction from $D+d$ to $\mathrm{D}$ dimensions results in a globally $O(d, d ; \mathbb{R})$ invariant theory in $D$ dimensions. It is assumed that the $(D+d)$ dimensional space-time can be written as a direct product of the form $\mathcal{M} \times K$, where $\mathcal{M}$ is a $D$ dimensional space-time with local coordinates $x^{\mu}(\mu=0,1, . ., D-1)$ and $K$ is a compact "internal" space with local coordinates $y^{\alpha}$ $(\alpha=1, . ., d)$. Furthermore it is assumed that all fields in the theory are independent of the internal coordinates $y^{\alpha}$. Now consider the action in $D+d$ dimensions

$$
\begin{aligned}
S & =\int_{N} d x \int_{K} d y \sqrt{-\hat{g}} e^{-\hat{\phi}}\left(\hat{R}(\hat{g})+\hat{g}^{\hat{\mu} \hat{\nu}} \partial_{\hat{\mu}} \hat{\phi} \partial_{\hat{\nu}} \hat{\phi}-\frac{1}{12} \hat{H}^{\hat{\mu} \hat{\nu} \hat{\lambda}} \hat{H}_{\hat{\mu} \hat{\nu} \hat{\lambda}}\right) . \\
\hat{\mu}, \hat{\nu} & =0, . ., D+d
\end{aligned}
$$

The hatted quantities now refer to the $(D+d)$-dimensional space-time. In order to see that the dimensionally-reduced action is invariant under global $O(d, d ; \mathbb{R})$ transformations it is important to introduce the following two quantities. First, the shifted dilaton which is invariant under $O(d, d ; \mathbb{R})$ transformations,

$$
\hat{\Phi} \equiv \hat{\phi}-\frac{1}{2} \log \operatorname{det} G_{\alpha \beta}
$$

Second, the following $2 d \times 2 d$ matrix $M$, written in $d \times d$ blocks,

$$
M \equiv\left(\begin{array}{lr}
G^{-1} & -G^{-1} B \\
B G^{-1} & G-B G^{-1} B
\end{array}\right)
$$

where $B$ denotes the matrix corresponding to the antisymmetric tensor field $B_{\alpha \beta}$, while $G$ is that for the internal metric $G_{\alpha \beta} . M$ is a symmetric $O(d, d)$ matrix. In terms of $M$, the low-energy action (11) can be written in an $O(d, d ; \mathbb{R})$ invariant way; that is, it is invariant under the transformations

$$
\hat{\Phi} \rightarrow \hat{\Phi} \quad M \rightarrow \Omega M \Omega^{T}
$$

where $\Omega \in O(d, d ; \mathbb{R})$.

Scale-factor duality, which was first described by [21], results when $G$ and $B$ are assumed to be only time-dependent.

\section{$3.2 S L(2, \mathbb{R})$ Symmetry}

In addition to the $O(d, d ; \mathbb{R})$ symmetry, which is a generalization of the discrete $O(d, d ; \mathbb{Z})$ T-duality group, there is also an $S L(2, \mathbb{R})$ symmetry which is a generalization of the discrete $S L(2, \mathbb{Z})$ S-duality group. 
Following [22], the $S L(2, \mathbb{R})$ of the equations of motion derived from the low-energy effective action can be revealed by first changing to the Einstein frame. First, we introduce a complex scalar field $\lambda$,

$$
\lambda=b+i e^{-\Phi}
$$

where $b$ is the axionic scalar field defined in (10). Writing the equations of motions in terms of $\lambda$ it can be verified that they are invariant under the transformation

$$
\lambda \rightarrow \lambda^{\prime}=\frac{\alpha \lambda+\beta}{\gamma \lambda+\delta} \quad \alpha \delta-\beta \gamma=1
$$

which is an $S L(2, \mathbb{R})$ transformation for $\alpha, \beta, \gamma, \delta \in \mathbb{R}$.

The strong-weak coupling interpretation of this $S L(2, \mathbb{R})$ symmetry emerges if the special element

with $\alpha=\delta=0$ and $\beta=1, \gamma=-1$ is considered. Applying this to a pure dilaton solution $\lambda=i e^{-\Phi}$ results in

$$
\lambda \rightarrow-\frac{1}{\lambda} \Leftrightarrow e^{-\Phi} \rightarrow e^{\Phi}
$$

Recalling that $e^{\Phi}$ can be interpreted as the string coupling, we see that this special transformation exchanges strong and weak coupling. As shown in [22] this symmetry of the equations of motion can also be extended to the low-energy effective action.

\section{Cosmological Solutions}

Solutions to the low-energy effective action have been found in different cosmological backgrounds. Friedmann-Robertson-Walker (FRW) backgrounds were discussed in [17]. The role of the antisymmetric tensor field strength was discussed in [23]. Inhomogeneous models were also investigated [24]. The solutions usually show an unbounded evolution of the dilaton, so the string coupling is not bounded. Furthermore, as in general relativity, curvature singularities are encountered although we expect higher-order terms to dominate in the vicinity of these ultra-high curvature regions.

\section{$5 \quad$ Pre-Big-Bang Cosmology}

The $O(d, d)$ (duality) symmetry inspired a new mechanism for inflationary evolution which does not rely on the potential energy of a scalar field as the source for inflation but on the combined action of dilaton and metric [15]. There are two branches which are related by duality. In the example discussed below, the $(+)$ branch is the pre-big-bang solution in which inflation occurs and to lowest order in the inverse string tension $\alpha^{\prime}$ the solution runs into a singularity at some time. The duality related (-) branch can be smoothly connected to a standard FRW exhibiting decelerated expansion with a constant dilaton. Then it is assumed that the dilaton potential becomes important and the dilaton settles down at its minimum, otherwise there might be problems with observations since a 
massless dilaton violates the equivalence principle [13] [16]. To satisfy observational constraints the dilaton mass has to be bounded below by 25 [16]

$$
M_{\Phi}>10^{-4} \mathrm{eV}
$$

In the standard big bang model the beginning of time coincides with the big bang. The pre-bigbang model dissolves this connection. The beginning of time is somewhere in the infinite past. The evolution from a weak coupling/low curvature regime to a high curvature regime provides conditions for joining the pre-big-bang to the post-big-bang stage in which the standard cosmological model (nucleosynthesis etc.) applies. This assumes the solution of the graceful exit problem which all the tree-level inflationary solutions have to face. In fact, in [26] it is shown that a smooth transition from the $(+)$ to the $(-)$ branch in the low-energy effective theory (that is, to lowest order in the inverse string tension $\alpha^{\prime}$ ), is not possible.

An attractive feature of the pre-big-bang model is that inflationary solutions follow naturally from the low-energy effective theory. The dilaton and its dynamics are given by the theory, so there is no need for assumptions of the kind made in standard inflationary models, where one has to justify the existence of a scalar field with a certain kind of potential.

A simple example of a pre-big-bang cosmology is set in a flat FRW background with a string frame metric

$$
d s^{2}=-d t^{2}+a^{2}(t) d x_{i} d x^{i} \quad i=1,2,3 .
$$

The dilaton satisfies $\Phi=\Phi(t)$ and for simplicity the antisymmetric tensor field strength is assumed to be vanishing. The solutions in pre- and post-big-bang regimes are given by [13] [26]

1. $(+)$ branch (defined in the domain $t<0$ )

$$
H=\mp \frac{1}{\sqrt{3} t} \quad \Phi=\Phi_{0}+(\mp \sqrt{3}-1) \ln |t|
$$

and $a$ evolves as $a=a_{0}\left(\frac{t}{t_{0}}\right)^{\mp \frac{1}{\sqrt{3}}}$.

2. (-) branch (defined in the domain $t>0$ )

$$
H= \pm \frac{1}{\sqrt{3} t} \quad \Phi=\Phi_{0}+( \pm \sqrt{3}-1) \ln t
$$

and $a$ evolves as $a=a_{0}\left(\frac{t}{t_{0}}\right)^{ \pm \frac{1}{\sqrt{3}}}$.

The solutions in the two domains are related by scale-factor duality, $a \rightarrow a^{-1}$, and time reflection, $t \rightarrow-t$. The $(+)$ branch solutions have a future singularity, that is they run into a singularity, whereas the $(-)$ branch solutions have a past singularity, that is they evolve from an initial singularity. It should be noted that these are the defining properties of the $(+)$ and $(-)$ branches. As pointed out in [26], the identification of the $(+)$ branch with the domain of negative times and the $(-)$ branch 
with that of positive times is just a coincidence. In general, this identification does not necessarily hold, especially when a dilaton potential is present.

The (-) branch describes either decelerated expansion, that is with $H>0$ and $\ddot{a}<0$; or accelerated contraction for $H<0$ and $\ddot{a}>0$. Choosing the expanding solution it can be shown that it can be smoothly connected to a standard FRW radiation-dominated solution [13]. Including in the equations of motion a matter source in form of a perfect fluid with energy density $\rho$, it turns out that the FRW radiation-dominated solution

$$
a=t^{\frac{1}{2}} \quad \Phi=\text { const. } \quad \rho=a^{-4}
$$

is a stable solution and it acts as an attractor for the dilaton of the $(-)$ branch solution [13].

In the $(+)$ branch domain there are solutions describing decelerated contraction $(H<0, \ddot{a}<0)$ and, more interestingly, solutions describing accelerated expansion $(H>0, \ddot{a}>0)$. These latter solutions describe so called super-inflationary models. Looking at the evolution of the dilaton in this case it can be seen that the string coupling $g_{s}^{2}$ evolves from very small values as $t \rightarrow-\infty$ to a strong coupling regime on approach to the "big bang" as $t \rightarrow 0$,

$$
g_{s}^{2}=e^{\Phi} \propto|t|^{-(\sqrt{3}+1)} .
$$

These solutions not only display inflationary behaviour driven by the kinetic energy of the dilaton but they also evolve from a weak coupling to a strong coupling regime. This means that the trivial, but exact, Minkowski solution of string theory could be taken as a starting point of the cosmological evolution. Furthermore, in the weak coupling regime the low-energy effective theory used here is perfectly valid.

This simple picture is modified once spatial curvature is admitted. The authors of ref. [27] investigated the impact of spatial curvature on the FRW pre-big-bang solutions. They found that the pre-big-bang inflationary solutions with positive or negative spatial curvature (i.e. $k$ positive or negative, respectively) require fine tuning of initial conditions in order to provide a long enough inflationary period. As described above, the flat pre-big-bang solutions can be, in principle, extended infinitely backwards in time while still describing a sensible cosmological model. However, the solutions with non-zero curvature differ from the flat ones. Solutions with positive curvature have an initial singularity. Those with negative curvature have a linearly increasing scale factor as $t \rightarrow-\infty$. So in the string frame the scale-factor starts with a large value at $t \rightarrow-\infty$ and subsequently decreases to some value $a_{\text {min }}$ before increasing up to the big bang singularity at $t=0$. In ref. 27] it was concluded that this behaviour can delay the onset of inflation and so the inflationary period might not be sufficient to solve the flatness and horizon problem. In ref. [28] it was argued that the problem with fine tuning in pre-big-bang inflationary models with spatial curvature is cured by the introduction of $\alpha^{\prime}$ corrections.

There are two types of corrections to the low-energy effective action: The string coupling $g_{s}^{2}$ determines the importance of string loop corrections and the inverse string tension $\alpha^{\prime}$ controls corrections due to the finite size of a string. Gasperini et al. [29] investigated the equations of motion in the case when the string coupling is still small, but $\alpha^{\prime}$ corrections become important. In this case the (four-dimensional) action reads [29] 28]

$$
S=-\frac{1}{2 \lambda_{s}^{2}} \int d^{4} x \sqrt{-g} e^{-\Phi}\left[R+(\nabla \Phi)^{2}-\frac{\alpha^{\prime}}{4}\left(R_{G B}^{2}-(\nabla \Phi)^{4}\right)\right]
$$


where $\lambda_{s}$ is the string length $\lambda_{s}^{2} \propto \alpha^{\prime}$ and

$$
R_{G B}^{2} \equiv R_{\mu \nu \alpha \beta}^{2}-4 R_{\mu \nu}^{2}+R^{2}
$$

is the Gauss-Bonnet term.

Considering the spatially-flat case, they found that the dilaton-driven super-inflationary stage with the scale-factor $a$ behaving as $a \propto(-t)^{-\frac{1}{\sqrt{3}}}$ is attracted to a state of exponential inflation and a linearly growing dilaton when the first-order $\alpha^{\prime}$ correction is included [29] [28]:

$$
a(t) \propto \exp \left(H_{s} t\right) \quad \Phi(t)=\Phi_{s}+c\left(t-t_{s}\right)
$$

with $H_{s}$ and $c$ constants. The constant $t_{s}$ is the time when the evolution enters the string phase, that is, when $\alpha^{\prime}$ corrections become important. So in this model there is a super-inflationary stage followed by a de Sitter stage, thereby regularizing the singularity which is unavoidable in the lowenergy string theory. Maggiore et al. [28] extended the arguments of [29] to include spatial curvature in the dilaton-driven stage and their numerical calculations show that in all three cases, i.e. for $k=0, \pm 1$, the first-order $\alpha^{\prime}$ correction regularize the singularity and lead to a stage of exponential inflation and linearly growing dilaton. They found that this additional inflationary stage can provide the required amount of inflation while still satisfying the various observational constraints.

\section{Gödel Universes}

Gödel's solution is at once an example of a homogeneous spacetime, a rotating universe, and an exact mathematical model of a world that contains closed timelike curves (CTCs). It provides a theoretical laboratory in which to study the existence and stability of some important global properties of spacetimes.

We write the general form of the string effective action to $\alpha^{\prime}$ order in the string frame as, [33, 38],

$$
\begin{aligned}
S=\int d^{n} x & \sqrt{-g} e^{-\phi}\left\{R-2 \Lambda+(\partial \phi)^{2}-\frac{1}{12} H^{2}\right. \\
& -\alpha^{\prime} \lambda_{0}\left[R_{\mu \nu \sigma \rho} R^{\mu \nu \sigma \rho}-\frac{1}{2} R^{\mu \nu \sigma \rho} H_{\mu \nu \alpha} H_{\sigma \rho}{ }^{\alpha}+\right. \\
& \left.\left.\frac{1}{24} H_{\mu \nu \lambda} H^{\nu}{ }_{\rho \alpha} H^{\rho \sigma \lambda} H_{\sigma}{ }^{\mu \alpha}-\frac{1}{8} H_{\mu \rho \lambda} H_{\nu}{ }^{\rho \lambda} H^{\mu \sigma \alpha} H^{\nu}{ }_{\sigma \alpha}\right]+O\left(\alpha^{\prime 2}\right)\right\}
\end{aligned}
$$

where $\lambda_{0}=-\frac{1}{8}$ for heterotic strings, $-\frac{1}{4}$ for bosonic strings and 0 for superstrings; $n$ is the number of spacetime dimensions, $\alpha^{\prime}$ is the inverse string tension parameter, $\phi$ is the dilaton, $g$ the determinant of the metric; $\Lambda$ is the cosmological constant, $R$ the Ricci scalar, $R_{\mu \nu \rho \sigma}$ the Riemann tensor, and $H_{\mu \nu \rho}$ is the axion with $H^{2}=H_{\mu \nu \rho} H^{\mu \nu \rho}$. The cosmological constant term $\Lambda$ is related to the dimension of space and the inverse string tension by $\Lambda=(n-26) / 3 \alpha^{\prime}$. This relation holds for bosonic strings; for superstrings $\Lambda$ is proportional to $n-10$.

Homogeneous spacetime metrics of Gödel type, [30], have the form 


$$
d s^{2}=-d t^{2}-2 C(r) d t d \psi+G(r) d \psi^{2}+d r^{2}+d z^{2},
$$

where the functions $C(r)$ and $G(r)$ have the forms

$$
\begin{aligned}
& C(r)=\frac{4 \Omega}{m^{2}} \sinh ^{2}\left(\frac{m r}{2}\right), \\
& G(r)=\frac{4}{m^{2}} \sinh ^{2}\left(\frac{m r}{2}\right)\left[1+\left(1-\frac{4 \Omega^{2}}{m^{2}}\right) \sinh ^{2}\left(\frac{m r}{2}\right)\right],
\end{aligned}
$$

with $m$ and $\Omega$ constants, [34]. In order to avoid the existence of CTCs in these spacetimes we require the no time-travel condition $G(r)>0$. This is consistent with the chronology protection conjecture of Hawking, [35].

Gödel universes rotate: the four-velocity of matter is $u^{\alpha}=\delta_{0}^{\alpha}$, the rotation vector is $V^{\alpha}=\Omega \delta_{0}^{3}$, and the vorticity scalar is given by $\omega=\Omega / \sqrt{2}$. The original Gödel metric of general relativity, [30], has $m^{2}=2 \Omega^{2}$ and clearly violates the no-time-travel condition. There has been extensive discussion about the generality and significance of the presence of CTCs in the Gödel metric in general relativity (see refs. [31], [32]).

The only nonvanishing components of the Riemann tensor in an orthonormal frame permitted by the spacetime homogeneity of the Gödel universe are constant, [36], with $R_{0101}=R_{0202}=\Omega^{2}, R_{1212}=$ $3 \Omega^{2}-m^{2}$. The dilaton depends only on the coordinate along the axis of rotation, $z$, so $\phi=\phi(z)=$ $f z+\phi_{0}$, where $f$ and $\phi_{0}$ are constants and $H_{012}=-H^{012}=E$, with $E$ constant. The field equations for the Gödel metric now reduce to the three polynomials. We now have three equations and six constants, leaving three (say, $\Lambda, f$ and $E$ ) arbitrary. We obtain $\Omega^{2}=m^{2} / 4$ which confirms the existence of a Gödel solution which fulfils the no time-travel condition. The value of $\alpha^{\prime}$ can now be expressed in terms of the velocity of rotation of the universe, $\Omega$, and the strength of axion field, $E$, giving [38],

$$
\alpha^{\prime}=\frac{E^{2}-4 \Omega^{2}}{4 \lambda_{0}\left(4 \Omega^{2}+E^{2}\right)\left(\Omega^{2}-\frac{5}{4} E^{2}\right)} .
$$

These are string theoretic generalisations of the famous rotating Gödel universes of general relativity. However, unlike in general relativity, string solutions exist which forbid time travel. These new solutions display remarkably simple relations between the rotation of the universe and the string tension by virtue of their $T$-duality symmetry. The simplicity of these relationships suggests that the remarkable symmetries of string theory may constrain peculiarities in the causal structure of spacetime that general relativity permits.

\section{Acknowledgements}

The authors would like to thank E. Copeland, M. Dąbrowski and J. Lidsey for discussions. JDB is supported by PPARC and KEK was supported by the German National Scholarship Foundation. 


\section{References}

[1] S.W. Hawking, R. Penrose , 1996, The Nature of Space and Time, Princeton University Press, Princeton.

[2] S.W. Hawking, G.F.R. Ellis, 1973, The large scale structure of space-time, Cambridge University Press, Cambridge.

[3] see for example, M. Kaku, 1988, Introduction to Superstrings, Springer Verlag, New York; M.B. Green, J.H. Schwarz, E. Witten , 1987, Superstring Theory, Volume 1, Cambridge University Press, Cambridge.

[4] M.J. Duff, 1996, M-Theory (The Theory formerly known as strings), hep-th/9608117; P.K. Townsend, 1996, in Proceedings of the 1996 ICTP Summer School in High Energy Physics and Cosmology (Trieste, June 10-26), hep-th/9612121.

[5] F. Quevedo, 1997, Superstring Phenomenology: An Overview, hep-ph/9707434.

[6] C.G. Callan, D. Friedan, E.J. Martinec, M.J. Perry, 1985, Nucl. Phys. B 262, 593.

[7] M. Giovannini, 1998, Phys. Rev. D 57, 7223.

[8] M. Gasperini, M. Maggiore, G. Veneziano, 1997, Nucl. Phys. B 494, 315; R. Easther, K. Maeda, 1996, Phys. Rev. D 54, 7252.

[9] R. Gueven, 1987, Phys. Lett. B 191, 275; G.T. Horowitz, A.R. Steif, 1990, Phys. Rev. Lett 64, 260; S. Kar, A. Kumar, G. Sengupta, 1996, Phys. Lett. B 375, 121.

[10] I. Antoniadis, C. Bachas, J. Ellis, D.V. Nanopoulos, 1988, Phys. Lett. B 211, 393; C. Nappi, E. Witten, 1992, Phys. Lett. B 293,309; see also G.T. Horowitz, D. Marolf, 1998, "A New Approach to String Cosmology", hep-th/9805207.

[11] see for example, E.W. Kolb, M.S. Turner, 1990, The Early Universe, Addison-Wesley, Redwood City, CA.

[12] R. Brustein, P.J. Steinhardt, 1993, Phys. Lett. B 302, 196; B.A. Campbell, A. Linde, K.A. Olive, 1991, Nucl. Phys. B 355, 146.

[13] R. Brustein, G. Veneziano, 1994, Phys. Lett. B 329, 429.

[14] T. Damour, A. Vilenkin, 1996, Phys. Rev. D 53, 2981.

[15] M. Gasperini, G. Veneziano, 1993, Astropart. Phys. 1, 317; G. Veneziano, 1998, "A simple/short introduction to pre-big-bang physics/cosmology", hep-th/9802057; for an updated collection of papers on string cosmology see http://www.to.infn.it/ gasperin.

[16] G. Veneziano, 1996, in Sánchez N., Zichichi A., eds, String Gravity and Physics at the Planck Energy Scale, Kluwer Academic Publisher, Dordrecht, The Netherlands. 
[17] E.J. Copeland, A. Lahiri, D. Wands, 1994, Phys. Rev. D 50, 4868.

[18] G. Veneziano, 1997, Phys. Lett. B 406, 297.

[19] J.A. Harvey, A. Strominger, 1992, Lectures given at 1992 Trieste Spring School on String Theory and Quantum Gravity; 1992 TASI Summer School in Boulder, Colorado, Quantum Aspects of Black Holes, hep-th/9209055; M. Gasperini, G. Veneziano, 1993, Mod. Phys. Lett. A 8, 3701.

[20] J. Maharana, J.H. Schwarz, 1993, Nucl. Phys. B 390, 3.

[21] G. Veneziano, 1991, Phys. Lett. B 265, 287; K.A. Meissner, G. Veneziano, 1991, Phys. Lett. B 267, 33.

[22] A. Sen, 1994, Int. J. Mod. Phys. A 9, 3707.

[23] N.A. Batakis, 1995, Phys. Lett. B 353, 39; N.A. Batakis, A.A. Kehagias, 1995, Nucl. Phys. B 449, 248; N.A. Batakis, 1995, Phys. Lett. B 353, 450; J.D. Barrow, K.E. Kunze, 1997, Phys. Rev. D 55, 623.

[24] J.D. Barrow, K.E. Kunze, 1997, Phys. Rev. D 56, 741; A. Feinstein, R. Lazkoz, M.A. VazquezMozo, 1997, Phys. Rev. D 56, 5166.

[25] T.R. Taylor, G. Veneziano, 1988, Phys. Lett. B 213, 450.

[26] N. Kaloper, R. Madden, K.A. Olive, 1995, Nucl. Phys. B 452, 677.

[27] M.S. Turner, E.J. Weinberg, 1997, Phys. Rev. D 56, 4604; see also N. Kaloper, A. Linde, R. Bousso, 1998, "Pre-Big bang requires the universe to be exponentially large from the very beginning", hep-th/9801073.

[28] M. Maggiore, R. Sturani, 1997, Phys. Lett. B 415, 335.

[29] M. Gasperini, M. Maggiore, G. Veneziano, 1997, Nucl. Phys. B 494, 315.

[30] K. Gödel, 1949, Rev. Mod. Phys. 21, 447; K. Gödel, In: Albert Einstein: Philosopher-Scientist, ed. P.A. Schilpp, (Open Court, Evanston, 1949).

[31] P. Nahin, Time Machines, (Springer-Verlag, NY, 1993); D.B. Malament, 1984, Proc. Phil. Sci. Assoc. 2, 91.

[32] S.W. Hawking and G.F.R. Ellis, The large scale structure of space-time,(Cambridge UP, 1973);

J. Earman, Bangs, Crunches, Whimpers, and Shrieks, (Oxford UP, 1995).

[33] R.R. Metsaev and A.A. Tseytlin, 1987, Nucl. Phys. B 293, 385.

[34] M.J. Rebouças and J. Tiomno, 1983, Phys. Rev. D 28, 1251.

[35] S.W. Hawking, 1992, Phys. Rev. D 46, 603. 
[36] A.J. Accioly, 1987, Nuovo Cim. B 100, 703.

[37] J.D. Barrow and M.P. Dąbrowski, 1997, Phys. Rev. D 55, 630.

[38] J.D. Barrow and M.P. Dąbrowski, 1998, "Gödel Universes in String Theory", gr-qc/9803048. 